\title{
Light propagation in highly scattering biological tissues analyzed by Green's functions
}

Jose L. Ganoza-Quintana, F. Fanjul-Vélez, J. L. ArceDiego

Jose L. Ganoza-Quintana, F. Fanjul-Vélez, J. L. Arce-Diego, "Light propagation in highly scattering biological tissues analyzed by Green's functions," Proc. SPIE 10876, Optical Interactions with Tissue and Cells XXX, 108760Q (1 March 2019); doi: 10.1117/12.2508963

SPIE. Event: SPIE BiOS, 2019, San Francisco, California, United States 


\title{
Light propagation in highly scattering biological tissues analyzed by Green's functions
}

\author{
J. L. Ganoza-Quintana, F. Fanjul-Vélez*, J. L. Arce-Diego* \\ Applied Optical Techniques Group, TEISA Department, University of Cantabria, Av de los Castros \\ $\mathrm{s} / \mathrm{n}, 39005$, Santander (Spain)
}

\begin{abstract}
Biomedical optical techniques of treatment, characterization and surgery are strongly dependent on light propagation in tissues. Information that goes beyond pure intensity, such as polarization or other coherence parameters, can provide increased contrast. This contrast is critical in clinical applications, as malignant tissue has to be distinguished from healthy one, or a particular component or structure has to be highlighted and detected. The appropriate consideration of these further light-tissue interaction properties requires taking into account phase and coherence. The complexity of the problem increases as biological tissues present usually high scattering. This fact greatly influences optical propagation, and is usually a fundamental limitation in optical diagnostic techniques. Light propagation in static scattering media can be analyzed by Green's functions. Electromagnetic propagation could be then considered, including coherence phenomena. However, analytical solutions are complex and require usually numerical methods to obtain a result. Monte Carlo approaches are particularly well-suited in biological tissues. In this work light propagation in highly scattering biological tissues is analyzed first by Green's functions. The limited geometry of this analytical approach serves as a first approach for more complex structures. More realistic biological tissue models are proposed and solved via a threedimensional time-resolved Monte Carlo approach. The model is applied to dermatological tumoral tissues. The results of scattering by Green's functions and the Monte Carlo approach are compared, and the potential contrast of coherence parameters is analyzed in diagnostic applications.
\end{abstract}

Keywords: Propagation of light, Scattering in biological tissues, Green's functions in optics, Optical properties, Numerical approaches for light-tissue interactions

\section{INTRODUCTION}

Biomedical optical techniques of treatment, characterization and surgery are strongly dependent on light propagation in tissues. Light propagation accuracy and a priori estimations are particularly significant in diagnostic techniques. Optical diagnostic techniques usually rely on intensity measurements, such as microscopy [1], fluorescence [2] or diffuse reflectance spectroscopy [3]. One way of further increasing contrast is the addition of polarization parameters, for instance in Optical Coherence Tomography [4]. In this last particular case information on phase and coherence is also of utmost relevance in the optical gating process, and could be also significant as additional contrast. This contrast is critical in clinical applications, as malignant tissue has to be distinguished from healthy one, or a particular component or structure has to be highlighted and detected. The appropriate consideration of these further light-tissue interaction properties requires taking into account phase and coherence. The complexity of the problem increases as biological tissues present usually high scattering. As biological tissue are turbid media, with a consequent great scattering influence, it is necessary to adequately analyze its influence on optical propagation parameters, such as coherence. The influence of a particular distribution of scatterers can be analyzed by Green's functions [5]. Electromagnetic propagation could be then considered, including coherence phenomena. However, analytical solutions are complex and require usually numerical methods to obtain a result. Monte Carlo approaches are particularly well-suited in biological tissues.

In this work light propagation in highly scattering biological tissues is analyzed first by Green's functions. The limited geometry of this analytical approach serves as a first approach for more complex structures. More realistic biological tissue models are proposed and solved via a three-dimensional time-resolved Monte Carlo approach. The model is applied to dermatological tumoral tissues. The results of scattering by Green's functions and the Monte Carlo approach are compared, and the potential contrast of coherence parameters is analyzed in diagnostic applications.

*fanjulf@unican.es; arcedj@unican.es; phone +34 94220 67 30; fax +34 9422018 73; www.teisa.unican.es/toa

Optical Interactions with Tissue and Cells XXX, edited by Hope Thomas Beier, Bennett L. Ibey, Proc. of

SPIE Vol. 10876, 108760Q - @ 2019 SPIE · CCC code: 1605-7422/19/\$18 · doi: 10.1117/12.2508963

Proc. of SPIE Vol. 10876 108760Q-1 
Next section 2 contains the theoretical model to analyze the light propagation in highly scattering biological tissues by Green's functions. Section 3 shows some of the results obtained and its discussion. Finally, section 4 includes the conclusions of the work.

\section{SCATTERING AND GREEN'S FUNCTIONS THEORETICAL MODEL}

One of the fundamental problems of field theory is the construction of solutions to linear differential equations when there is a specified source and the differential equation must satisfy certain boundary conditions. Green's functions provide a method for obtaining these solutions. Let $\boldsymbol{F}_{L d}[f(x)]=g(x)$ be a linear differential operator plus certain boundary conditions for $\mathrm{f}(\mathrm{x}), \mathrm{BC}[\mathrm{f}(\mathrm{x})]$ and represented in the usual Signals and Systems scheme (considering onedimensional signals and derivative operators). In a physical problem, usually $\mathrm{g}(\mathrm{x})$ is known - source of some physical magnitude - while $f(x)$ is the magnitude generated by $g(x)$. This leads directly to the definition of the Green's function as the set of functions (distributions in a rigorous way) which are the set of impulse responses of the inverse system $\boldsymbol{F}_{L \boldsymbol{d}, \boldsymbol{B} C}^{-1}[g(x)]=f(x)$. These functions, denoted by $\mathrm{G}\left(\mathrm{x} ; \mathrm{x}^{\prime}\right)$, have to satisfy the Green's function problem,

$$
\left\{\begin{array}{c}
\boldsymbol{F}_{\boldsymbol{L d}, \boldsymbol{B C}}\left[G\left(x ; x^{\prime}\right)\right]=\delta\left(x-x^{\prime}\right) \\
B C\left[G\left(x ; x^{\prime}\right)\right]
\end{array}\right\} \rightarrow f(x)=\int_{x} g\left(x^{\prime}\right) G\left(x ; x^{\prime}\right) d x^{\prime}
$$

Scattering in biological media represents one of the most limiting effects for optical diagnostic imaging. Along with light intensity, coherence is particularly affected by the scattering mechanism [6]. The scattered wave $U_{s}(r, \omega)$ from a monochromatic incident wave $U_{i}(r, \omega)$ can be expressed as a total $U(r, \omega)$ wave, assuming the first-order Born approximation, as:

$$
U(r, \omega) \sim U_{i}(r, \omega)+\int_{D} F\left(r^{\prime}, \omega\right) U_{i}\left(r^{\prime}, \omega\right) G\left(\left|r-r^{\prime}\right|, \omega\right) d^{3} r^{\prime}
$$

In equation (2), $F(r, \omega)$ is the scattering potential of the medium, and $G\left(\left|r-r^{\prime}\right|, \omega\right)$ is the outgoing free-space Green function. If incident light is partially coherent, as it is a more general case, with a cross-spectral density function $W_{i}\left(r_{1}, r_{2}, \omega\right)$, the cross-spectral density of the scattered wave can be expressed as:

$$
W_{s}\left(r_{1}, r_{2}, \omega\right)=\iint_{D} W_{i}\left(r_{1}^{\prime}, r_{2}^{\prime}, \omega\right) F^{*}\left(r_{1}^{\prime}, \omega\right) F\left(r_{2}^{\prime}, \omega\right) G^{*}\left(\left|r_{1}-r_{1}^{\prime}\right|, \omega\right) G\left(\left|r_{2}-r_{2}^{\prime}\right|, \omega\right) d^{3} r_{1}^{\prime} d^{3} r_{2}^{\prime}
$$

The cross-spectral density can be expressed as a function of the spectral densities $S_{i}\left(r_{1}^{\prime}, \omega\right)$ and $S_{i}\left(r_{2}^{\prime}, \omega\right)$, and the spectral degree of coherence $\mu_{i}\left(r_{1}^{\prime}, r_{2}^{\prime}, \omega\right)$ :

$$
W_{s}\left(r_{1}, r_{2}, \omega\right)=\iint_{D} \sqrt{S_{i}\left(r_{1}^{\prime}, \omega\right)} \sqrt{S_{i}\left(r_{2}^{\prime}, \omega\right)} \mu_{i}\left(r_{1}^{\prime}, r_{2}^{\prime}, \omega\right) F^{*}\left(r_{1}^{\prime}, \omega\right) F\left(r_{2}^{\prime}, \omega\right) G^{*}\left(\left|r_{1}-r_{1}^{\prime}\right|, \omega\right) G\left(\left|r_{2}-r_{2}^{\prime}\right|, \omega\right) d^{3} r_{1}^{\prime} d^{3} r_{2}^{\prime}
$$

The coherence properties can be also expressed in the space-time domain by the mutual coherence function:

$$
\Gamma_{s}\left(r s_{1}, r s_{2}, \tau\right)=\frac{1}{r^{2}} \int_{0}^{\infty} S_{i}(\omega) \widetilde{F^{*}}\left[k\left(s_{1}-s_{0}\right), \omega\right] \tilde{F}\left[k\left(s_{2}-s_{0}\right), \omega\right] e^{-i \omega \tau} d \omega
$$

Although this analysis may lead to a coherence propagation solution, it is only analytically solvable in limiting cases of low complexity. Biological tissues require usually numerical methods, such as the Monte Carlo approach. The use of 3D meshes to represent the tissue overcomes further limitations and allows to depict complex heterogeneous structures. The Mesh-based Monte Carlo (MMC) [7] is based in a Monte Carlo method that employs a 3D tetrahedral mesh to represent the biological tissues and a Plücker coordinate ray tracing algorithm. It allows to obtain the light propagation both time resolved and in steady state. The direction of the photon beam can be determined by means of two points, $p\left(x_{1}, y_{1}, z_{1}\right)$ and $q\left(x_{2}, y_{2}, z_{2}\right)$. Plücker coordinates allow to express a 3D beam by means of vectors displacement $d=q-p$ and moment $m=p \times q$. The main advantage of using this coordinate system is the ease that it offers to calculate the intersections beam-polygon, by evaluating the expression:

$$
w_{i}=U_{r} V_{e i}+V_{r} U_{e i}
$$

Where the beam $r\left(U_{r}, V_{r}\right)$ enters $\left(w_{i}>0\right)$, leaves $\left.\left(w_{i}<0\right)\right)$ or is coplanar to $\left.\left(w_{i}=0\right)\right)$ the surface of the triangle with vertices $\left(U_{e 1}, V_{e 1}\right),\left(U_{e 2}, V_{e 2}\right),\left(U_{e 3}, V_{e 3}\right)$. This approach can be used to coherence propagation [8]. 


\section{RESULTS AND DISCUSSION}

The analysis of the previous section was applied to a model of dermatological tissue. In particular, the model includes an inclusion of tumoral tissue, in this example Squamous Cell Carcinoma (SCC). Figure 1 shows some results of spatiallyresolved light distribution as a function of beam impinging position. The tumoral area is defined by the gray rectangle in the images.
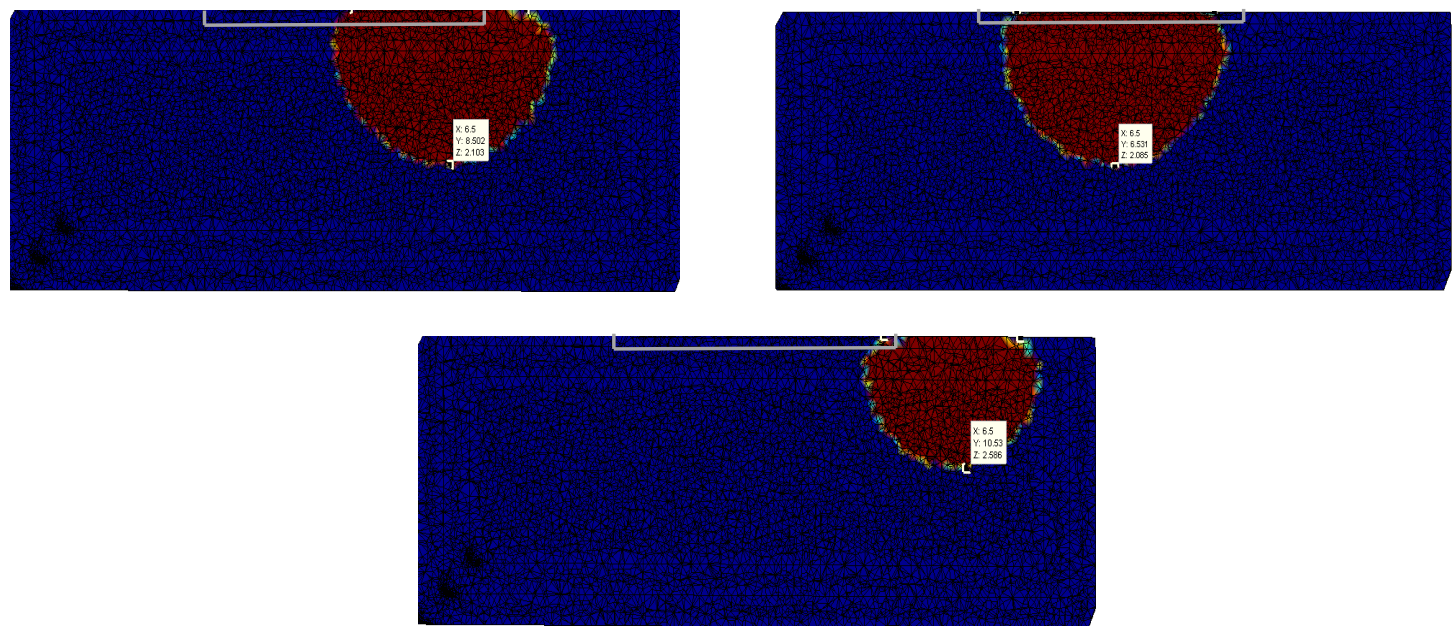

Fig.1. Model of SCC tumor (grey rectangle at the tissue surface) in epithelial tissue. The optical source is impinging on the central area (upper left), displaced $2 \mathrm{~mm}$ (upper right) and at $4 \mathrm{~mm}$ (lower image).

The combination of the scattering approach by Green's functions, and that of the coherence properties on Monte Carlo three-dimensional and time-resolved numerical methods could be of great interest in biomedical optical diagnostic applications.

\section{CONCLUSIONS}

Biomedical optical techniques of treatment, characterization and surgery are strongly dependent on light propagation. Further parameters based on polarization or coherence can provide increased contrast in diagnostic techniques. Light propagation in static highly scattering biological tissues can be analyzed by Green's functions, including coherence phenomena. Analytical solutions are complex and require usually numerical methods, such as Monte Carlo approaches. Light propagation in highly scattering simplified media has been analyzed first by Green's functions. Realistic models have been solved via three-dimensional Monte Carlo. Results for dermatological tumoral tissues were obtained, and the potential diagnostic contrast of coherence parameters was analyzed.

\section{ACKNOWLEDGEMENTS}

This work has been partially supported by the project "New active phases in transition metals and rare earth nano-oxides stabilized at high pressure" (MAT2015-69508-P) of the Spanish Ministry of Economy and Competitiveness, cofunded by FEDER funds, and by the San Cándido Foundation.

\section{REFERENCES}

[1] Girkin, J. M. and Carvalho, M. T., “The light-sheet microscopy revolution,” J. Opt. 20, 053002 (2018).

[2] Salas-García, I., Fanjul-Vélez, F. and Arce-Diego, J. L., "Superficial radially-resolved fluorescence and threedimensional photochemical time-dependent model for Photodynamic Therapy," Opt. Lett. 39, 1845-1848 (2014). 
[3] Fanjul-Vélez, F., Arévalo-Díaz, L. and Arce-Diego, J. L., "Intra-class variability in diffuse reflectance spectroscopy: application to porcine adipose tissue," Biomedical Optics Express 9, 2297-2303 (2018).

[4] Fanjul-Vélez, F., Pircher, M., Baumann, B., Götzinger, E., Hitzenberger, C. K. and Arce-Diego, J. L., "Polarimetric analysis of the human cornea by Polarization Sensitive Optical Coherence Tomography," J. Biomed. Opt. 15, 056004 (2010).

[5] Ganoza-Quintana, J. L., Fanjul-Vélez, F. and Arce-Diego, J. L., "Optical coherence propagation in biological tissues with significant scattering by Green's functions," Optical Society of America, Frontiers in Optics, paper JTu3A.106 (2018).

[6] Born, M. and Wolf, E., Principles of Optics (Cambridge University Press, 2013), Chap.13.

[7] Fang, Q., "Mesh-based Monte Carlo method using fast ray-tracing in Plücker coordinates," Biomedical Optics Express 1, 165-75 (2010).

[8] Shen, Z., Sukhov, S. and Dogariu, A., "Monte Carlo method to model optical coherence propagation in random media," J. Opt. Soc. Am. A 34, 2189-2193 (2017). 